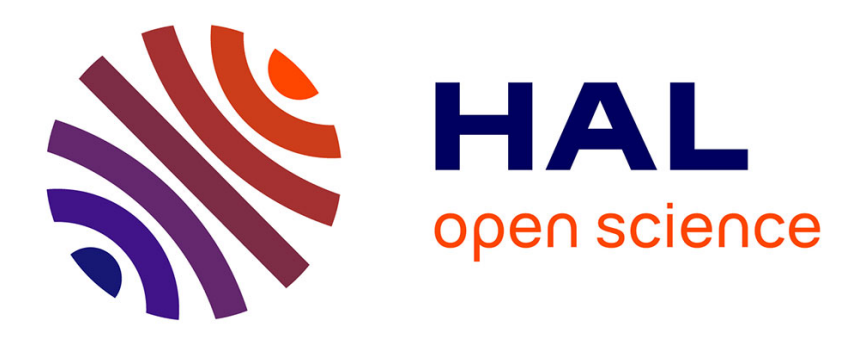

\title{
Fonder les villes : comment les terrassiers comprennent le sol
}

Germain Meulemans

\section{To cite this version:}

Germain Meulemans. Fonder les villes : comment les terrassiers comprennent le sol. Communications, 2019, 105 (2), pp.149-159. 10.3917/commu.105.0149 . hal-02882980

\section{HAL Id: hal-02882980 \\ https://hal.science/hal-02882980}

Submitted on 28 Jun 2020

HAL is a multi-disciplinary open access archive for the deposit and dissemination of scientific research documents, whether they are published or not. The documents may come from teaching and research institutions in France or abroad, or from public or private research centers.
L'archive ouverte pluridisciplinaire HAL, est destinée au dépôt et à la diffusion de documents scientifiques de niveau recherche, publiés ou non, émanant des établissements d'enseignement et de recherche français ou étrangers, des laboratoires publics ou privés. 


\title{
Fonder les villes : comment les terrassiers comprennent le sol
}

\author{
Germain Meulemans
}

Ce texte est la version finale acceptée d'un article publié le 04/11/2019 dans Communications, $\mathrm{n}^{\circ} 105$, pp. 149-159. https://doi.org/10.3917/commu.105.0149

La construction des fondations est rarement considérée comme une activité créative, au même titre que l'architecture ou l'urbanisme. Si les historiens de l'architecture réservent la part belle aux voûtes en ogive et autres structures métalliques permettant des formes toujours plus aériennes, ils ne parlent que rarement de sols ou de fondations ${ }^{1}$. Tout, dans l'épopée de l'architecture, semble renvoyer à une émancipation progressive vis-à-vis du sol, qui est presque toujours représenté comme une page blanche sur laquelle matérialiser les projets humains ${ }^{2}$. Le sol apparaît ainsi comme un socle purement matériel pour le déploiement de la vie, de l'histoire et des idées émergeant à sa surface.

Le peu de cas réservé au sol dans l'histoire de l'architecture et de l'urbanisme se rapporte à une plus large séparation entre ce qui se dresse au-dessus de la surface de la terre et ce qui se trame en dessous, entre le monde physique du sol et le monde aérien des idées. Pour l'anthropologue Tim Ingold, ces traits sont caractéristiques de la pensée moderne de la ville : «Les pieds sur terre et la tête en l'air, les êtres humains semblent constitutionnellement divisés entre le matériel et le mental ${ }^{3}$. » L'étymologie du mot «sol » est elle-même liée à celle de l'adjectif « solide », du latin solidus, « massif, compact, consistant » et, au sens figuré, « ferme, inébranlable $»^{4}$. Encore aujourd'hui, le sol évoque un sentiment de solidité, de compacité, d'indivision et de fiabilité. Il se laisse ainsi oublier comme l'arrière-plan par trop matériel des constructions humaines. Dans cet article, nous nous proposons de questionner l'idée de sol comme page blanche, en nous intéressant aux fondations et à ceux qui les construisent. Nous nous basons sur un terrain ethnographique réalisé auprès des techniciens et ingénieurs de la branche parisienne d'une grande entreprise de géotechnique entre février et mars 2015, enquête poursuivie par plusieurs visites de chantiers et par des entretiens menés avec des terrassiers, géologues et ingénieurs des carrières entre 2015 et 2018.

La mission des terrassiers et des géotechniciens est de rendre les sols stables. Ce sont eux qui préparent le terrain avant même que l'architecte se mette à l'ouvrage, et c'est grâce à leur travail - et à son invisibilité - que l'idée même du site comme une terra nullius et appropriable est possible. Comme tant d'autres activités communément affublées du qualificatif de «technique », la géotechnique a pour tâche de mettre les sols en « boîte noire $»^{5}:$ du moment que fondations et terrassements sont bien 
réalisés, personne ne peut soupçonner qu'un sol puisse être autre chose qu'une assise solide et pleine. Terrassiers et géotechniciens nous emmènent pourtant dans un monde bien différent de celui de la ville surfacique du cadastre et de la carte. Pour eux, le sol est une entité hétérogène et mouvante qui brouille la limite entre solide et fluide. Le sol devient ce socle appropriable par une série d'opérations techniques, et son caractère solide relève donc d'une forme de " performativité ${ }^{6}$ ». Contrairement au français, les langues germaniques distinguent le sol en tant que terre fertile (soil en anglais), du sol en tant que masse ou surface de la Terre (ground). C'est bien du second qu'il est question ici. Parler de sol, pour un terrassier c'est faire référence à des couches et des poches d'argiles, de limons, de graviers et autres matériaux géologiques, parfois rapportés par l'homme, tous composés de grains de tailles et de formes différentes.

À cause du caractère local et émergent des propriétés des sols, les ingénieurs ne peuvent déduire leur comportement à partir d'un modèle mathématique établi a priori. Par une série d'opérations que nous décrirons, en reprenant les travaux de l'anthropologue Stefan Helmreich, comme des opérations d' « abduction », il leur faut approcher le comportement d'immenses couches de sol à partir d'un engagement tactile et auditif avec une poignée de grains seulement, et inventer ou « rencontrer » sans cesse des solutions pratiques pour s'ajuster aux propriétés inattendues des sols. À travers leurs activités, la notion même d'inertie du sol urbain ne se donne plus tant à voir comme une simple absence de mouvements, mais invite à être comprise dans sa dimension pragmatique ${ }^{7}$ - celle d'une lente et hasardeuse solidification à travers nombre de détours techniques. Ainsi, comme le remarquait déjà Karl Terzaghi ${ }^{8}$, l'un des fondateurs de la mécanique des sols, les fondations sont faites en « vivant avec les sols », en accordant une attention constante aux forces qui les traversent et en jouant avec leur équilibre fragile plus qu'en les modelant suivant un dessein extérieur préétabli.

\section{S'ancrer en territoire semi-fluide.}

L'histoire moderne de l'ingénierie des fondations - sous ses diverses appellations de mécanique des sols ou de géotechnique - est marquée par une controverse irrésolue autour de la nature même des sols, et de la possibilité et de l'utilité d'établir une théorie générale et prédictive de leur comportement. Du XVIIIe au milieu du XXe siècle, des ingénieurs cherchèrent à établir une théorie générale du comportement du sol vis-à-vis des constructions ${ }^{9}$. L'objectif était d'isoler des lois universelles permettant de constituer des bases rationnelles pour la construction des fondations. De telles théories avaient pu être établies pour d'autres matériaux, facilitant ainsi leur utilisation à grande échelle. Comme le note Reint de Boer, des matériaux tels que la céramique ou le béton avaient pu être traités comme des matériaux « idéaux », pleins et apparemment dépourvus de structure interne : «avec de tels modèles, la mécanique des milieux continus classique avait pu obtenir des résultats très probants ${ }^{10}$. Cependant, les sols posèrent rapidement un problème particulier : devaient-ils être 
compris comme des fluides, ou comme des solides ? Les théoriciens ne purent jamais caractériser les sols de manière définitive, les regardant tour à tour comme des solides, comme des fluides imparfaits, ou comme des semi- fluides. De plus, les avancées théoriques les plus notoires - telles que celles de Charles-Augustin Coulomb à la fin du XVIIIe siècle - ne purent se faire qu'à partir d'un sol si abstrait et épuré qu'elles ne reçurent que peu d'échos auprès des constructeurs ${ }^{11}$. Le débat entre solidité et fluidité, ou entre théorie et expérience, se retrouve ailleurs à la même époque, autour de sujets tels que le mouvement des glaciers ${ }^{12}$. Il semble partout difficile aux modélisateurs de réconcilier fluidité et solidité, deux propriétés envisagées comme des attributs des matériaux, et qui semblent difficiles à penser ensemble.

En opposition aux ambitions de la théorisation, on trouve à chaque époque des ingénieurs défendant une approche pratique, considérant le sol comme un matériau si complexe et spécifique à chaque lieu et à chaque projet que ses propriétés ne peuvent jamais être entièrement saisies par la théorie. Dès le XIXe siècle, on renvoie donc l'ingénieur au terrain : «À lui de faire de son chantier un laboratoire où il occupera ses loisirs ${ }^{13}$. »C C'est ainsi qu'en 1957, Karl Terzaghi, l'ingénieur autrichien tenu pour être le fondateur de la mécanique des sols moderne, affirmait que, « pour acquérir des compétences dans le domaine du terrassement, il faut véritablement vivre avec le sol. Il faut l'aimer et observer son comportement non seulement en laboratoire mais aussi sur le terrain, afin de se familiariser avec les multiples propriétés que ne peuvent prendre en compte les modèles ennuyeux ${ }^{14}$ ». Face aux lois mathématiques, abstraites, ne se voulant ancrées nulle part et supposées vraies partout, on voit donc s'affirmer la primauté de l'expérience, du terrain et de l'observation. Les géotechniciens que nous avons pu rencontrer mentionnent fréquemment l'attention, et même la passion, qu'ils développent envers les vices et vertus multiples et toujours locaux des sols avec lesquels ils ont à travailler. Ils disent parfois que leur métier est lié à un «terroir » particulier. Ici, il s'agit du Bassin parisien, constitué d'une grande diversité de couches géologiques dans ses cinquante premiers mètres. Formées par les cycles de compression et de relâchement de la croûte terrestre, le dépôt de sédiments marins, ou les aléas des méandres et affluents de la Seine, ces couches ont chacune des propriétés différentes, et les fondations de bâtiments traversent souvent plusieurs d'entre elles. Ainsi, les ingénieurs savent que les couches profondes constituées d'alluvions de la Seine déposées lors des dernières glaciations sont souvent tourbeuses et peuvent s'affaisser, alors que les « anciennes alluvions » sont quant à elles assez vieilles pour avoir eu le temps de se tasser. Comme nous 1'explique l'un d'eux : «Ici à Paris, nous connaissons nos argiles, nous connaissons nos limons... Quand je parle avec des entrepreneurs sur un chantier, nous n'utilisons pas le vocabulaire de la géotechnique, mais il suffit de parler de "la couche verte", et nous savons tous ce que cela signifie. » Il affirme parlà l'importance de l'expérience d'un lieu, de ses couches et des matériaux dont il est fait. S'il était amené à réaliser une étude dans un endroit différent, il ne saurait pas aussi facilement à quoi s'attendre. 
Les fondations qui seront réalisées dépendent à la fois de ce terroir et du savoir-faire local. À Paris, on creuse le plus souvent jusqu'au « sol dur » qu'offrent les couches de roche calcaire pour y poser les fondations. Parfois, on se contente d'enfoncer des pieux d'acier ou de béton (autrefois, ceux-ci étaient en bois) pour y ancrer le bâtiment. Dans les régions où le sol est particulièrement instable, comme dans les zones soumises à des tremblements de terre fréquents, les fondations tendent à n'être pas ancrées dans la roche mère, mais reposent plutôt sur un radier - une large plateforme de béton armé placée sous terre. Ainsi, en cas de mouvement du sol, l'ensemble du bâtiment, incluant ses fondations, se déplacera de manière solidaire - comme un bateau qui tangue sur une mer agitée, en quelque sorte.

\section{Entendre le sol sécher : l'abduction du granulaire.}

Les paysages souterrains auxquels s'intéressent les géotechniciens sont généralement accessibles par l'excavation uniquement. Cependant, avant même que toute machine de creusement soit amenée sur le site, les propriétés des sols sont évaluées à partir de petites quantités échantillonnées à diverses profondeurs grâce à des carottages.

Dès que le technicien ouvre le sac ou la gaine d'un échantillon, il le regarde et le touche pour en ressentir la texture, l'homogénéité ou l'hétérogénéité. Il joue avec l'échantillon dans sa main, modifie sa forme, essaie de repérer les changements de couleur et de texture, et le déplace sous la lumière pour mieux en apprécier le grain. Il cherche à tout prix à identifier des traces de briques ou de béton. Celles-ci indiqueraient un sol de remblais, remanié par l'homme, dont on ne peut supposer l'homogénéité et dont le comportement est donc trop imprévisible - un tel sol sera presque toujours excavé et envoyé en décharge. Au laboratoire, comme sur le chantier, le géotechnicien est particulièrement attentif à la façon dont les grains crissent et grincent. Cette attention au son des grains en friction n'est pas anodine. Pour Salomé Voegelin ${ }^{15}$, l'expérience du son est celle d'un monde de relations. Le son que font les grains de sol dit quelque chose de la manière dont ils se rencontrent. L'engagement de la peau et de l'oreille avec les grains ouvre aux particularités relationnelles de ces derniers, à la façon dont ils se lient ensemble, s'agencent ou se défont aussitôt qu'ils sèchent ou se gonflent d'eau.

Divers tests permettent de séparer les ingrédients du sol et d'établir des classes de grains. La cohésion du sol dépend principalement des types de matériaux qui le composent et de leur degré d'humidité. Certains matériaux, comme l'argile, deviennent solides et pratiquement cohérents une fois secs. D'autres, comme le sable, sont incohérents lorsque secs, et durs lorsque humides. Ces tests permettent ainsi d'examiner le comportement des sols en fonction des teneurs en humidité d'échantillons broyés en poudre, séchés, puis humidifiés pour observer le gonflement. Les mesures liées à l'humidité fournissent ainsi les informations les plus précieuses sur les caractéristiques du sol et permettent d'évaluer ses capacités à se comporter 
comme un solide, un liquide ou une matière plastique. Ainsi, pour un ingénieur rencontré pendant notre enquête, «l'eau est à la base de tout ce qui peut mal tourner ». L'eau fait gonfler certains sols, stagne dans d'autres, les fait parfois glisser ou s'affaisser, ou en dissout certains composants. Les sols en pente, comme ceux situés sur le versant des collines, peuvent être entraînés sous leur propre poids lorsqu'ils se gorgent d'eau. Bien que cela produise parfois des glissements de terrain spectaculaires, ces déplacements dus au travail de l'eau sont la plupart du temps quasiment imperceptibles, mais non moins dommageables à long terme pour les bâtiments dont les fondations sont lentement mises en désordre.

Le travail par lequel l'ingénieur approche le comportement des immenses couches de sol à partir de la façon dont quelques milliers de grains se comportent dans de petits appareils de test et crissent dans la main peut être décrit comme un processus d'abduction. Ce concept a d'abord été défini par Charles Sanders Peirce comme « une méthode permettant de former une pré- diction générale sans assurance positive que cette prédiction sera vérifiée ». Pour Peirce, il s'agirait d'un mode de réflexion plus créatif que l'induction, mais qui n'interviendrait qu'en dernier recours, lorsqu'il reste « le seul espoir possible de réguler une conduite future rationnellement ${ }^{16}$. Cette définition a été reprise et précisée par l'anthropologue Stefan Helmreich ${ }^{17}$ qui, dans sa description du travail de microbiologistes confrontés aux étranges formes de vie des profondeurs sous-marines, combine la définition de Peirce avec le sens plus général d'abduction en anglais : celui d'un rapt, d'un enlèvement. Pour lui, les microbiologistes qui s'aventurent dans les profondeurs des mers sont comme capturés par les formes de vie qu'ils y rencontrent, et auxquelles ils ne peuvent donner sens avec leurs référentiels habituels : «Les humains, étrangers dans le monde marin, emploient un mélange de logique et "d'intuition de la dernière chance" afin de donner du sens à l'inconnu ${ }^{18}$. " L'abduction est différente de l'induction, car elle ne déduit pas de conclusion générale à partir d'observations particulières. Alors que l'induction opère à partir d'éléments supposés être des « faits » dans un certain cadre épistémologique, l'abduction s'ancre dans la complexité d'un état présent, une complexité que l'on ne peut capturer totalement. La limite floue, dans la notion d'abduction, entre le mode de pensée et le rapt permet aussi d'insister sur l'idée que l'étrangeté de l'objet implique de se laisser affecter par lui ${ }^{19}$ : puisqu'on ne peut délimiter clairement le matériau sol $a$ priori il ne reste qu'à le suivre là où il mène ${ }^{20}$.

Aussi, le riche vocabulaire servant à décrire les sols implique souvent un devenir plutôt qu'une qualité intrinsèque, des effets, des actions possibles et des problèmes probables. Par exemple, la tourbe peut être fibreuse, pseudo-fibreuse ou amorphe. Elle peut également être ferme, spongieuse, plastique, noire, marron ou blonde, et à divers stades de décomposition. Pour le géotechnicien, la description de ces éléments est ancrée dans le présent du matériau, mais renvoie dans le même temps à ses états futurs - non pas à une prédiction du comportement du sol, mais à des potentialités ou dangers éventuels, qui devront être reliés aux situations de chantier. Un ingénieur nous explique ainsi que les tests en laboratoire ne sont qu'un moyen de voir « ce que ce sol a dans le ventre », une 
métaphore digestive qui fait référence à la fois à ce dont le sol est fait et à ce qu'il peut faire. Ce que les géotechniciens cherchent à sonder à travers leur observation attentive, la manipulation et les tests des sols, ce sont les potentialités qui pourraient se manifester dans l'interaction entre les couches de grains instables, des flux d'eau erratiques, le poids du bâtiment, les propriétés de ses matériaux et la gravité terrestre. Pour reprendre une distinction de Tim Ingold ${ }^{21}$, ils ne sont pas surpris par un monde qui ferait mentir leurs prédictions, mais le prennent comme une source continue d'étonnement. Les géotechniciens sont entrainés à s'attendre à la variation, et plutôt que de chercher à prédire le comportement du sol, il s'agit pour eux d'observer et d'anticiper ses changements.

\section{De l'imprévisibilité à l'étrangeté du sol urbain.}

Nous pourrions être enclins à penser qu'en perdant le sol à travers la traduction qu'effectuent les tests de laboratoire, nous avons acquis des connaissances sur celui$\mathrm{ci}^{22}$. Cependant, les géotechniciens savent très bien que la «traduction » du sol en une série de mesures de granulométrie et de teneur en eau permet de se faire une idée approximative des propriétés du sol remis dans son contexte. Même quand ils connaissent bien les propriétés particulières de leur «terroir », les ingénieurs géotechniciens savent que le sol peut être un compagnon difficile. Il faut toujours garder un œil sur cette masse imprévisible et puissante.

Prenons l'exemple du chantier d'un bâtiment situé à proximité du boulevard périphérique de Paris, que nous avons visité avec l'ingénieure en charge de la construction des fondations. Elle a planifié les travaux de terrassement et de consolidation sur la base des « hypothèses de sol », issues des prospections réalisées sur le site et des tests de laboratoire. Elle sait cependant que ses plans doivent ensuite être adaptés localement, car des parties du sol environnant réagissent toujours différemment de ce qui est prévu. Il est donc important de surveiller ce qui se passe dans le sol et dans le bâtiment « au fur et à mesure qu'ils vivent leur vie ». Elle supervise donc la mise en œuvre des travaux de fondation, et reviendra contrôler l'ouvrage une fois les travaux achevés.

Quelques jours auparavant, alors que les ouvriers creusaient, le fond de la fouille a commencé à se remplir d'eau. De telles inondations sont très courantes sur les chantiers à l'intérieur des villes, et elles peuvent rarement être prévues. Comme nous l'explique cette ingénieure :

Il y a des endroits où il y a de l'eau, d'autres où il n'y en a pas. À la campagne, on rencontre plutôt de grandes nappes phréatiques. Nous savons à peu près où elles sont et nous adaptons les plans dès le départ. Mais on ne sait jamais à quoi s'attendre lorsqu'on creuse en ville. Le sol est perturbé par les constructions, l'eau pénètre dans les jardins, les terrains de football, puis elle se trouve 
bloquée à cause des constructions. Il peut donc y avoir des poches d'eau n'importe où.

Ainsi, les poches d'eau sont piégées entre des obstacles imperméables - tels que les fondations d'autres bâtiments - et sont libérées lorsque le creusement de nouvelles fondations leur ouvre un passage. De telles fuites sont également dangereuses pour l'ensemble des bâtiments situés autour du site d'excavation. En s'écoulant, l'eau entraîne les particules les plus fines des sols qu'elle traverse. Ceux-ci risquent donc de se tasser, provoquant désordres et fissures dans les bâtiments qu'ils soutiennent. Si un tassement du sol entraine des dommages sur un tuyau d'eau et qu'une fuite se déclare, le problème peut se retrouver multiplié de façon exponentielle. C'est pourquoi, lorsque des désordres sont constatés sur un bâtiment (si les habitants constatent des fissures ou, dans les cas plus graves, ne peuvent plus fermer leur porte), les techniciens qui sont appelés pour rechercher un éventuel tassement du sol commencent toujours par rechercher une fuite d'eau qui serait passée inaperçue, et s'interrogent sur la présence de chantiers dans les environs. Comme l'explique cette ingénieure, « dans tous les types de terrassements, nous impactons le sol et son fonctionnement, de sorte que nous pouvons toujours incidemment provoquer, couper ou réorienter des flux d'eau ». Même en rase campagne, la construction peut entraver un chemin d'écoulement préférentiel des eaux de pluie, ou endommager d'anciens drains agricoles oubliés dont la destruction entraine des inondations. Le terrassement est donc un double combat contre le mouvement des sols : s'il vise à ancrer les bâtiments dans un sol stable, la construction des fondations provoque des vides créateurs de mouvements, et tout travail sur la surface a des conséquences sous ou dans la zone environnante. L’imprévisibilité du sol vient du fait que celui-ci, n'existant pas ex nihilo comme dans un laboratoire, interagit continuellement avec le climat, les bâtiments environnants et la fouille en elle-même. Les propriétés du sol sont donc contextuelles, et même émergentes. Il en va de lui comme d'autres matériaux, dont les propriétés « ne sont pas des attributs mais des histoires ${ }^{23}$ ». On ne peut apprécier sa solidité ou sa fluidité a priori, ces qualités n'étant pas dans la nature du sol, car elles émergent de l'intrication du sol et de son environnement. Puisque le caractère solide ou fluide du sol ne peut être apprécié qu'en situation, dans le déroulement continu des relations entre le sol et son environnement, l'art de la construction des fondations en appelle à l'expérience du constructeur, dans la double acception du terme - les acquis, sa maîtrise du «terroir géotechnique »-, mais aussi dans le sens phénoménologique du tact, du toucher, du ressenti à partir de quantités infimes de sol. Il s'agit par-là d'approcher le sol pris dans son devenir, par une série d'opérations que nous avons caractérisée comme une forme d'abduction.

\section{Conclusion : vivre avec les sols.}

Ce rapide tour d'horizon des pratiques des constructeurs de fondations permet de mieux comprendre le sens du mot de Terzaghi selon lequel ces praticiens doivent « 
vivre avec les sols » pour bien faire leur métier. Le sol des terrassiers est mouvement : ses matériaux vivent dans une écologie matérielle de dilution, d'érosion, de compression et de dissolution. Ils glissent, s'effondrent et ne sont jamais tout à fait solides. Comme le montre l'exemple du fond de fouille inondé, les constructions humaines ne se trouvent pas simplement posées sur le sol de la ville, elles en deviennent partie intégrante en modifiant l'équilibre en jeu entre ses forces compositionnelles, et, de ce fait, ne peuvent que participer à l'intrication complexe de leurs flux. Dès lors, contrairement à la plupart des habitants des villes, terrassiers et géotechniciens doivent prêter attention non pas à la seule surface du monde, mais plutôt à l'interaction entre les courants souterrains et les bâtiments qui, avec un peu d'imagination, semblent presque flotter dedans ${ }^{24}$. Leur manière de « vivre avec les sols » nous invite donc à questionner la compréhension commune de la ville en tant qu'entité épigée, se développant sur la surface stable de la terre. Elle permet de contredire l'idée d'un monde qui serait par nature un arrière-plan matériel à l'histoire humaine, et de montrer que l'épaisseur du sol ne peut être oubliée qu'au prix d'un patient travail de construction et de stabilisation, tant matériel que symbolique. S'intéresser à la surface des choses en tant que réalité contingente permet d'attirer l'attention vers la performativité des formes de stabilité et de matérialité qui nous entourent, ainsi que vers les types d'expériences sensorielles et politiques du monde auxquelles elles donnent lieu. Les sols des villes ne sont qu'un cas parmi un vaste répertoire de surfaces qui partitionnent le monde des modernes, et qui va de la peau, surface du corps, à l'écran tactile d'un téléphone ${ }^{25}$. Une attention large portée aux nombreuses occurrences de formes de pensée «surfaciques » dans les sociétés modernes peut constituer un point d'entrée original et fécond pour l'étude des manières dont les frontières entre matérialité et créativité, entre le monde et l'esprit, ou entre nature et culture, prennent consistance.

\section{$\underline{\text { Notes }}$}

${ }^{1}$ Panos Mantziaras, «Le sol des villes, un projet critique pour l'anthropocène », in Panos Mantziaras et Paola Viganò (dir.), Le Sol des villes. Ressource et projet, Genève, MētisPresses, 2016, p. 7-16.

${ }^{2}$ Germain Meulemans et Tibo Labat, « Le chantier comme enquête », in ibid., p. 145-158.

${ }^{3}$ Tim Ingold, Being Alive : Essays on Movement, Knowledge and Description, Londres, Routledge, 2011, p. 74 (nous traduisons).

${ }^{4}$ Félix Gaffiot, Dictionnaire illustré latin-français, Paris, Hachette, 1934, p. 1453.

${ }^{5}$ Comme le montre Stephen Graham, le travail continu qu'implique la maintenance des infrastructures tend à rester invisible pour leurs utilisateurs. Il ne revient sur le devant de la scène que lorsqu'un ouvrage se fend ou s'effondre. Stephen Graham, Disrupted Cities: When Infrastructure Fails, New York, Routledge, 2010.

${ }^{6}$ Bronislaw Szerszynski, Heim Wallace et Claire Waterton, Nature Performed : Environment, Culture and Performance, Oxford, Blackwell, 2003. 
${ }^{7}$ Theodoros Kyriakides, « Stillness as a Form of Imaginative Labour », The Unfamiliar, 4 (1), 2014.

${ }^{8}$ Karl Terzaghi, « Opening session at the Institution of Civil Engineers, Monday, 12 August, at 2.30 P.M. », Proceedings of the International conference on Soil Mechanics and Foundation Engineering, 4 (3), 1957, p. 5558 , p. 58 (nous traduisons).

${ }^{9}$ André Guillerme, « La cervelle de la terre : la mécanique des sols et les fondations d'ouvrage de 1750 à 1830 », History and Technology, 7 (3-4), 1991, p. 211-254 ; Sabine Barles, La Ville délétère. Médecins et ingénieurs dans l'espace urbain xviiie-xixe siècle, Seyssel, Champ Vallon, 1999, p. 156-164.

${ }^{10}$ Reint de Boer, «Highlights in the Historical Development of the Porous Media Theory : Toward a Consistent Macroscopic Theory », Applied Mechanics Reviews, 49 (4), 1996, p. 201-262, p. 201.

${ }^{11}$ André Guillerme, « La cervelle de la terre », art. cité, p. 217.

${ }^{12}$ Cristián Simonetti et Tim Ingold, «Ice and Concrete : Solid Fluids of Environmental », Journal of Contemporary Archaeology, 5 (1), 2018, p. 19-31.

${ }^{13}$ André Guillerme, « La cervelle de la terre », art. cité, p. 222.

${ }^{14}$ Karl Terzaghi, « Opening session at the Institution of Civil Engineers, Monday, 12 August, at

2.30 P.M. », op. cit., p. 58 (nous traduisons).

${ }^{15}$ Salomé Voegelin, Sonic Possible Worlds. Hearing the Continuum of Sound, Londres/New York, Bloomsbury, 2014. Voir dans ce sens le commentaire de l'ouvrage par Thibault De Meyer, Lectures, 2015, mis en ligne le 22 septembre 2015 : http://journals.openedition.org/lectures/18937

${ }^{16}$ Charles Sanders Peirce, A Syllabus of Certain Topics of Logic, 1903, manuscrit non publié (EP 2:299), cité in Stefan Helmreich, Alien Ocean : Anthropological Voyages in Microbial Seas, Berkeley, University of California Press, 2009, p. 230 (nous traduisons).

${ }^{17}$ Stefan Helmreich, «Induction, Deduction, Abduction, and the Logics of Race and Kinship »,

American Ethnologist, 34, 2007, p. 230-232 ; id., Alien Ocean, op. cit.

${ }^{18}$ Ibid., p. 194.

${ }^{19}$ On se rapprocherait alors du mode d'enquête caractérisé par Evelyn Fox Keller, A Feeling for the Organism : The Life and Work of Barbara McClintock, San Francisco, W. H. Freeman, 1983.

${ }^{20}$ Ailleurs, nous avons développé une analyse du creusement comme manière de « suivre le sol » à partir de l'étude des pratiques de creusement de fosses en pédologie. Germain Meulemans, « Des hommes qui creusent : suivre le sol en pédologie » in Léo Mariani et Carine Plancke (dir.), (D)écrire les affects, Paris, Petra, 2018, p. 299-325 ; Voir aussi l'article pionnier de Matt Edgewoth sur la manière dont les archéologues suivent l'histoire de la pointe de leur truelle. Matt Edgeworth, «Follow the Cut, Follow the Rhythm, Follow the Material», Norwegian Archaeological Review, 45(1), 2012, p. 76-92.

${ }^{21}$ Tim Ingold, Being Alive, op. cit., p. 74-75.

${ }^{22}$ Bruno Latour, «Le "pédofil" de Boa Vista - montage photo-philosophique », in Petites leçons de sociologie des sciences, Paris, La Découverte/Le Seuil, 1996, p. 171-225. 
${ }^{23}$ Tim Ingold, Being Alive, op. cit., p. 32.

${ }^{24}$ Une promenade en ville lors d'un jour venteux a inspiré une observation similaire à Ingold. Tim Ingold, « What if the city were an ocean, and its buildings ships ? », Livingmaps Review, 2, 2017, p. 1-5.

${ }^{25}$ Pour d'autres exemples, voir le champ en pleine expansion des surface studies, auquel le site www.surfacestudies.org constitue une bonne introduction, ou encore l'ouvrage collectif de Mike Anusas et Cristián Simonetti (dir.), Surfaces : Transformations of Body, Materials and Earth, Londres, Routledge, à paraitre 2020. 